\title{
Conocimiento y actitud de médicos residentes de pediatría sobre caries dental en niños, Paraguay
}

\section{Knowledge and management of Pediatrician interns on dental caries in children, Paraguay}

\section{Resumen}

Objetivo: Determinar el conocimiento y la actitud acerca de la prevención de caries dental en el niño, en médicos residentes de la especialidad troncal de pediatría, de la XI y XVIII regiones sanitarias (Departamento Central y Asunción) del Paraguay, en el año 2015. Materiales y método: Estudio de diseño transversal. Se administró un cuestionario solicitándose datos demográficos y académicos, el cuestionario contó con 10 preguntas sobre conocimiento de caries dental y seis preguntas sobre actitud. Resultados: Participaron 98 médicos residentes de la especialidad de Pediatría. Más de la mitad fueron egresados de universidades privadas (53,06\%), procedieron de Asunción $(65,31 \%)$ y fueron mujeres $(68,37 \%)$. El $83,67 \%$ tuvo conocimiento insuficiente sobre prevención de caries dental, el 16,33\% obtuvo conocimiento regular y ninguno obtuvo conocimiento bueno. El 96,94\% mostró una actitud positiva, el 3,16\% una actitud indiferente y ninguno tuvo actitud negativa acerca del papel del pediatra en la prevención de la caries dental. Conclusiones: En la mayoría de médicos residentes, el conocimiento fue insuficiente y la actitud positiva. A tal efecto, desde la formación académica se debería incentivar la promoción y prevención sobre salud bucodental del infante como corresponsabilidad del pediatra.

Palabras clave: Caries dental; Internado y residencia; Paraguay; Pediatría.
ISSN: $1560-9111$

Artículo Original

Clarisse Díaz-Reissner ${ }^{1, a, e}$, María Quintana-Molinas 1,a, Milner Morel-Barrios 1,a,c, Miriam Espínola-Canata ${ }^{2, b, e}$, Nohelia Pérez-Bejarano ${ }^{1, a, d}$

${ }^{1}$ Facultad de Odontología. Universidad Nacional de Asunción. Asunción, Paraguay.

${ }^{2}$ Instituto Nacional de Salud. Ministerio de Salud Pública y Bienestar Social. Asunción, Paraguay. a Odontólogo.

${ }^{\mathrm{b}}$ Médico. Especialista en Pediatría.

${ }^{c}$ Especialista en Salud Pública y Administración Hospitalaria.

${ }^{d}$ Especialista en Odontopediatría.

e Máster en Metodología de la Investigación en Ciencias de la Salud.

Correspondencia:

Clarisse Díaz-Reissner

Correo electrónico: cdiazr@odo.una.py

Avda. España c/ Brasil. Asunción-Paraguay.

\section{Coautores:}

María Quintana-Molinas

maria.e90una@hotmail.com

Milner Morel-Barrios

morelbarrios1980@gmail.com

Miriam Espínola-Canata

mecanata@gmail.com

Nohelia Pérez-Bejarano

dra.nohe@gmail.com

Conflicto de intereses: Los autores declaran no tener conflictos de interés.

Fuente de financiamiento: Autofinanciado.

Fecha de recepción: 11/09/17

Fecha de aceptación: 25-09-17

\begin{abstract}
Objective: To determine knowledge and attitude on child dental caries prevention among pediatric interns of XI and XVIII sanitary regions (Central and Asuncion Departments) of Paraguay, during 2015. Materials and method: Transversal study. A questionnaire was administered requiring both demographic and academic data, with 10 questions on dental caries knowledge and six question on attitude. Results: Ninety-eight Pediatric interns participated in the study. More than half of participants graduated from private universities (53.06\%), they were from Asunción (65.31\%), and 68.37\% were women. Insufficient knowledge regarding dental caries prevention was found in $83.67 \%, 16.33 \%$ had fair knowledge and none had thorough knowledge. A positive attitude was showed by $96.94 \%$, indifferent attitude was found in $3.16 \%$ and none showed a negative attitude about the role on preventing dental caries that pediatricians play. Conclusions: The majority of pediatric interns in question; showed insufficient knowledge yet a positive attitude. Consequently, a more structured oral health prevention curriculum should be enforced during academic instruction as a shared responsibility with pediatricians.
\end{abstract}

Keywords: Dental caries; Internship and residency; Paraguay; Pediatrics.

(C) Los autores. Este artículo es publicado por la revista Odontología Sanmarquina de la Facultad de Odontología, Universidad Nacional Mayor de San Marcos. Este es un artículo de acceso abierto, distribuido bajo los términos de la licencia Creative Commons Atribucion - No Comercia_Compartir Igual 4.0 Internacional. (http://creativecommons.org/licenses/by-nc-sa/4.0/) que permite el uso no comercial, distribución y reproducción en cualquier medio, siempre que la obra original sea debidamente citada. 


\section{Introducción}

En Latinoamérica, generalmente son limitados los recursos para apoyar programas de salud oral, registrándose de esta manera una brecha entre la prevención y los tratamientos necesarios, el resultado implica que los nińos estén propensos a un alto riesgo de desarrollar caries durante sus años preescolares ${ }^{1}$. La persistencia de caries dental no tratada en la dentición primaria de niños de edad escolar sigue siendo un problema de salud pública, con una prevalencia del $70 \%$ en países en desarrollo ${ }^{2,3}$.

Paraguay tiene un "CPO-D intermedio" a la edad de 12 años, es decir, un promedio de tres dientes cariados por niño cuando la dentición permanente recién se ha completado, lo que evidencia la necesidad de reforzar las políticas de prevención y promoción de salud bucal en el sistema de salud nacional ${ }^{4}$. Este escenario obliga a implementar o fortalecer los programas de prevención enfocados en la higiene oral efectiva en niños desde los primeros ańos de vida y las orientaciones sobre la dieta adecuada, brindando la información relacionada a la madre desde el embarazo, acerca de los cuidados dentales del bebé, debido a que resulta más efectivo prevenir hábitos indeseados que eliminarlos una vez instaurados 5 .

El pediatra es el primer profesional médico en examinar a los niños, es el que mantiene el contacto más frecuente y hasta puede llegar a establecer un vínculo entre el niño y su entorno, por lo que es crucial su rol motivador para la conservación de la salud bucal de los mismos ${ }^{6}$. Mínimamente, debería recomendar la primera visita al odontopediatra a los seis meses de edad, tal como lo sugiere la Asociación Americana de Odontología ${ }^{7}$.

La prevalencia de caries dental para los niños más pequeños no ha disminuido en la última década. Los datos de una encuesta del panel de gastos médicos revelaron que el $89 \%$ de bebés y niños de un año, anualmente representan el 1,5\% de las visitas dentales. En consecuencia, las visitas a los médicos superaron a las visitas a los dentistas en 250 a 1 para este grupo de edad ${ }^{8}$ por lo que el impacto de la prevención y la gestión de las enfermedades orales requiere la atención de profesionales de la salud y tomadores de decisiones y se extiende mucho más allá del consultorio dental ${ }^{9}$. Una vez ocurrido este contacto, el odontopediatra deberá proporcionar tratamiento dental e influir en el comportamiento futuro de la salud bucal del niño, de manera que la prevención sea una prioridad a través de un examen bucal, un plan de tratamiento, y el establecimiento del mecanismo de comunicación efectiva con el pediatra ${ }^{10}$.

Las enfermedades bucales por definición, se refieren a las condiciones de los dientes, las encías y la boca, e incluyen entre otras a la caries dental. El impacto de estas condiciones sobre la calidad de vida es alto, común y de tratamiento costoso, por lo que se consideran un problema de salud pública de alta relevancia. Estas enfermedades están socialmente modeladas, afectando desproporcionadamente a poblaciones socialmente desfavorecidas y marginadas. Debido a que la salud oral y la salud general están inextricablemente vinculadas y comparten factores de riesgo comunes, son indicadores útiles de la salud en general y patrones generales de desigualdades en salud ${ }^{11}$.

El daño de la salud oral en el niño puede ocurrir en varios niveles como señala el resultado de un estudio que afirma que la caries no tratada en etapas avanzadas se asoció con una peor calidad de vida entre los nińos de uno a tres años y sus familias ${ }^{12}$. La calidad de vida relacionada con la salud bucodental es un concepto multidimensional relacionado con el impacto que una mala salud o enfermedad oral tiene sobre el funcionamiento diario, el bienestar o la calidad de vida de un individuo y su entorno ${ }^{13}$. La caries dental no tratada en niños puede ser una de las causas de ausentismo escolar, un problema con impacto económico para su familia que puede interferir además con sus actividades diarias como el juego, descanso, una correcta alimentación, entre otros problemas que podrían resumirse en factores que afectan el desarrollo del niño, su aprendizaje, la funcionalidad familiar, las cargas económicas que impone, así como también a las comunidades y el sistema de salud ${ }^{9}$.

En el año 2008 de 9151 médicos registrados en el Ministerio de Salud Pública y Bienestar Social (MSPyBS) del Paraguay, el 8,23\% eran pediatras ${ }^{14}$. Anualmente, se recibieron en la especialidad troncal de pediatría aproximadamente 67 médicos. Los mismos realizan su residencia médica en las XVII, X, XI y XVIII Regiones Sanitarias, en los Hospitales Pediátricos Niños de Acosta $\mathrm{Nu}$, Central del Instituto de Previsión Social, General de Barrio Obrero, Regional de Luque, Nacional de Itaguá, Regional de Encarnación, Regional de Ciudad del Este y en el Instituto de Medicina Tropical; encontrándose todos estos hospitales e institutos reconocidos por la Comisión Nacional de Residencias Médicas (CONAREM) como unidades formadoras en dicha área ${ }^{15,16}$. Cabe mencionar, que en el país existen dieciocho regiones sanitarias, establecidas por el MSPyBS ${ }^{17}$.

Un estudio realizado en Paraguay en el año 2013, con el objetivo de determinar el nivel de conocimiento, actitud y práctica sobre prevención en salud bucal, realizado en 92 pediatras de Asunción y el Departamento Central, concluyó que el nivel de conocimiento sobre prevención de caries dental en la primera infancia fue aceptable en menos de la mitad de los participantes, siendo las actitudes acordes con la importancia de prevención de enfermedades bucodentales en la totalidad y la práctica inadecuada en la mayoría de ellos ${ }^{18}$. No existen estudios similares, por lo que resulta un tema poco ahondado y estudiado en nuestro medio.

Por lo expuesto, considerando que los pediatras son los primeros en tomar contacto con el niño y su familia, resulta relevante que estén bien preparados y motiven a la consulta odontológica desde temprana edad. Teniendo en cuenta que la formación de los pediatras como especialistas se inicia en los hospitales donde realizan la residencia médica, se consideró oportuno indagar desde los inicios de su formación en el área, cuales son los conocimientos adquiridos y las actitudes acerca de su 
responsabilidad en el manteamiento de la salud bucal del niño. Se planteó como objetivo del estudio determinar el nivel de conocimiento y la actitud acerca de la prevención de la caries dental en el infante, en médicos residentes de la especialidad troncal de pediatría de la XI y XVIII regiones sanitarias del Paraguay, en el año 2015.

\section{Materiales y Método}

El diseño fue transversal ${ }^{19}$. La población quedó constituida por médicos residentes de la especialidad primaria o troncal de pediatría del primer, segundo y tercer año, cuyas unidades formadoras forman parte del listado del Programa Nacional de Residencias por la Comisión Nacional de Residencias Médicas (CONAREM) ${ }^{20,21}$ órgano dependiente del Ministerio de Salud Pública y Bienestar Social, Asunción, en el año 2015. El protocolo de investigación fue aprobado por el Comité de Ética en Investigación del Hospital Central del Instituto de Previsión Social. El cuestionario fue anónimo, se mantuvo la confidencialidad de los datos obtenidos.

Para una precisión del 7\% en la estimación de una proporción mediante un intervalo de confianza para una distribución normal, con corrección para poblaciones finitas al 95\% bilateral, asumiendo que la proporción esperada de conocimiento suficiente es del 49\% basado en el estudio de Rolón-Lara et al. ${ }^{18}$ en pediatras paraguayos y que el tamaño total de la población fue de 174, se incluyó a 98 sujetos en el estudio. Teniendo en cuenta que el porcentaje esperado de cuestionarios incompletos fue del $10 \%$, fue necesario reclutar 104 sujetos. Se solicitó permiso al Departamento de Educación Médica, Docencia e Investigación del Hospital Central del Instituto de Previsión Social y a los jefes de servicios de Pediatría de cada hospital o instituto dependientes de la Universidad Católica Nuestra Señora de la Asunción; excepto el Hospital Nacional de Itauguá que no depende de dicha universidad, al cual se le solicitó autorización de manera separada. Todos los residentes fueron invitados a participar del estudio. Los cuestionarios fueron entregados a los jefes de cada servicio y recogidos al mes. El cuestionario se aplicó de manera autoadministrada.

Se utilizó el cuestionario validado de Rojas-Mayhuire 22, aplicado en médicos pediatras del Colegio Médico de Perú, adaptado en lo referente a aspectos culturales por dos odontólogos paraguayos. El mismo contiene 10 preguntas de selección múltiple con una respuesta correcta acerca de conocimiento sobre etiología, diagnóstico y tratamiento, prevención y repercusión sistémica de la caries dental en niños. Para evaluar el conocimiento se consideró tres niveles: Insuficiente [0 a 4 respuestas correctas], Regular [5 a 7 respuestas correctas] y Bueno [8 a 10 respuestas correctas]. Para evaluar la actitud se realizaron seis preguntas con opciones de respuesta en escala Likert de cinco categorías que fueron desde "totalmente de acuerdo" a "totalmente en desacuerdo" acerca de la responsabilidad del pediatra en la prevención de caries dental en el niño. Para evaluar la actitud se consideró tres categorías: Negativa [0 a 10 puntos], Indiferente [1 11 a 20 puntos] y Positiva [21 a 30 puntos]. Además, se incorporaron ítems acerca de datos demográficos y académicos.

Se utilizó el programa Ene 3.0@ de Glaxo Kline Smith para el cálculo del tamaño de la muestra. Para el análisis de datos se utilizó estadística descriptiva mediante el programa EpiInfo 7.1.4 de la Center of Disease Control and Prevention (CDC). Se reportó la media y desviación estándar para variables cuantitativas, frecuencias y porcentajes para variables cualitativas. Se aplicó la prueba Chi-cuadrado de Pearson para evaluar la homogeneidad de la distribución del nivel de conocimiento con variables sociodemográficas, con un nivel de confianza del $95 \%$.

\section{Resultados}

Participaron del estudio 98 médicos residentes de la especialidad de pediatría del Hospital Central del Instituto de Previsión Social, Hospital General de Barrio Obrero, Hospital Regional de Luque, Instituto de Medicina Tropical y Hospital Nacional de Itauguá, en el año 2015. Más de la mitad fueron egresados de universidades privadas, la mayoría de los encuestados procedió de Asunción y fueron mujeres (Tabla 1). La edad promedio fue de $27 \pm 1,9$ años con un mínimo de 23 años y un máximo de 36 .

Tabla 1. Características demográficas y académicas de los médicos residentes de Pediatría, Paraguay 2015.

\begin{tabular}{|c|c|}
\hline Variable & $\%$ \\
\hline \multicolumn{2}{|l|}{ Año de residencia } \\
\hline $1^{\text {ero }}$ & 28,57 \\
\hline $2^{\mathrm{do}}$ & 48,98 \\
\hline $3^{\text {ero }}$ & 22,45 \\
\hline \multicolumn{2}{|l|}{ Hospital/Instituto } \\
\hline Previsión Social & 51,02 \\
\hline Barrio Obrero & 21,43 \\
\hline Luque & 6,12 \\
\hline Itauguá & 16,33 \\
\hline Medicina Tropical & 5,1 \\
\hline \multicolumn{2}{|l|}{ Universidad de egreso } \\
\hline Pública & 46,94 \\
\hline Privada & 53,06 \\
\hline \multicolumn{2}{|l|}{ Procedencia } \\
\hline Asunción & 65,31 \\
\hline Gran Asunción & 24,49 \\
\hline Interior del país & 10,2 \\
\hline \multicolumn{2}{|l|}{ Sexo } \\
\hline Femenino & 68,37 \\
\hline Masculino & 31,63 \\
\hline
\end{tabular}


El 83,67\% demostró conocimiento insuficiente sobre caries dental, el 16,33\% conocimiento regular y ninguno obtuvo el conocimiento esperado como "bueno", no habiéndose encontrado relación con el sexo $(\mathrm{p}=0,971)$, la universidad de egreso $(\mathrm{p}=0,408)$, año de residencia $(\mathrm{p}=0,578)$ y procedencia $(\mathrm{p}=0,752)$.

Un bajo porcentaje respondió que no sabía cuál es la principal bacteria causante de caries dental, más de la mitad no asoció el factor hereditario con el desarrollo de caries dental en el infante, la misma proporción respondió correctamente que las bebidas gaseosas son cariogénicas, un poco más de la mitad contestó correctamente que la caries avanzará hacia la dentina si no es tratada y un elevado porcentaje conocía que el antibiótico de primera elección para el tratamiento de infecciones odontogénicas producidas por caries es la amoxicilina (Tabla 2).

Un elevado número de participantes desconocía que la higiene bucal debe realizarse desde el nacimiento y la edad y tipo de aplicación de flúor, pero la mitad sabía que la primera consulta odontológica debe realizarse entre los 6 meses y 1 año (Tabla 3).

El 96,94\% tuvo una actitud positiva, el 3,16\% actitud indiferente y ninguno actitud negativa acerca del papel del pediatra en la prevención de la caries dental. La mayoría coincidió en que el pediatra debe ser el principal proveedor de información y consejero sobre salud oral en niños, además que debe examinar los dientes y diagnosticar caries dental en las consultas de control del niño sano, que estaría dispuesto a examinar la cavidad bucal, proporcionar información sobre salud bucal y derivar al odontopediatra (Tabla 4).

\section{Discusión}

El nivel de conocimiento acerca de la prevención de caries dental en el niño fue insuficiente en la mayoría de encuestados, mientras que la actitud demostrada hacia dicho tema fue positiva en la mayoría de los médicos residentes de la especialidad troncal de pediatría que participaron de esta encuesta. Este resultado sugiere la necesidad de un refuerzo en el programa curricular tanto de grado como de posgrado, con énfasis del área estomatológica, teniendo en cuenta que no se encontró diferencias en cuanto al nivel de conocimientos por año de residencia.

Una publicación sobre una encuesta similar realizada en pediatras de La India reveló que el 39,7\% tenía conocimiento moderado, $36,5 \%$ buen conocimiento y $23,8 \%$ bajo conocimiento sobre caries dental ${ }^{23}$. Mientras que en el estudio realizado en Paraguay se encontró que el conocimiento fue inadecuado en más de la mitad de los pediatras. En el mismo sentido, en pediatras andaluces (España) se encontró que el conocimiento fue escaso y que los mismos no informaban detalladamente a los padres acerca de cuidados de salud oral ni orientaban hacia la necesidad de visitas al odontopediatra ${ }^{6}$. En médicos recién recibidos que realizaban internado rotatorio en un hospital-escuela en Teherán encontraron que la edad, el sexo y los años de formación no tuvieron efecto en el nivel de conocimiento, siendo el conocimiento general bajo ${ }^{24}$. En nuestro estudio un elevado porcentaje tuvo conocimiento insuficiente considerando que las coincidencias en los resultados de estudios locales y externos exigen un planteamiento de estudios que busquen las razones de este fenómeno.

En ese sentido, en Arabia Saudita, en un estudio se concluyó que los estudiantes de grado deberían tener una mayor exposición a pacientes con problemas de salud oral para mejorar su experiencia, además de mejorar el conocimiento a través de la formación, centrándose estos esfuerzos en aquellos que elegirán especialidades clínicas orientadas al área de salud pública, ya que ha demostrado ser un factor importante asociado a la posibilidad de realizar diversas prácticas de salud oral en los niños pequeños ${ }^{25}$. En cuanto a la actitud, los pediatras de La India se mostraron con predisposición muy favorable hacia un rol más activo en la prevención de caries en la mayoría de los encuestados $(81,8 \%)$, dicha actitud positiva denotó aun mayor compromiso en los pediatras paraguayos de este estudio, quienes en su totalidad estuvieron de acuerdo en que deberían cumplir un papel importante en la prevención de enfermedades bucales ${ }^{23}$.

Rolón Lara et al. ${ }^{18}$ sugieren que se deben formular programas de formación en salud bucodental en pediatras paraguayos para paliar los déficits resultantes no solo en el ámbito de la salud física, sino también en las posibles repercusiones de la esfera psicosocial que pudieran afectar al niño en su etapa de desarrollo; en el mismo sentido, según el estudio hecho en Teherán, también surge la necesidad de recomendar la incorporación en los planes de estudios contenidos curriculares acerca de salud oral en los médicos ${ }^{24}$. Ambos coinciden con los resultados obtenidos en nuestro estudio, donde un elevado porcentaje tuvo una actitud positiva.

Es importante resaltar que una vez incorporados en los contenidos curriculares aspectos sobre salud oral del infante y el impacto de esta en el equilibrio del individuo y su entorno, se convertirían en capacidades de los pediatras, las cuales se traducirían en oportunas derivaciones a la consulta dental temprana, pues está demostrada la existencia de bajas tasas de referencia para los niños con enfermedad y antes de la aparición de la enfermedad, pero con un riesgo elevado, por lo que es necesaria la implementación de intervenciones para mejorar las tasas de derivación dental de los médicos ${ }^{26}$.

\section{Conclusiones}

En la mayoría de médicos encuestados el conocimiento sobre caries dental fue insuficiente y la actitud fue positiva. A tal efecto, desde la formación académica se debería incentivar la promoción y prevención sobre salud bucodental del infante como corresponsabilidad del pediatra. 
Tabla 2. Conocimientos sobre caries dental en niños. Médicos Residentes de Pediatría, Paraguay 2015.

\begin{tabular}{|c|c|}
\hline Variable & $\%$ \\
\hline \multicolumn{2}{|l|}{ Factores asociados a la caries dental } \\
\hline Defectos del esmalte & 2,05 \\
\hline Herencia & 60,2 \\
\hline El amamantamiento frecuente y prolongado & 27,55 \\
\hline Ingestión frecuente de alimentos ricos en carbohidratos & 4,08 \\
\hline Trauma dentoalveolar* & 3,06 \\
\hline No sabe & 3,06 \\
\hline \multicolumn{2}{|l|}{ Principal bacteria causante de la caries dental } \\
\hline Streptococcus oralis & 15,31 \\
\hline Streptococcus salivarius & 21,43 \\
\hline Streptococcus mutans* & 40,82 \\
\hline Lactobacillus acidoufilus & 7,14 \\
\hline Ninguna de las anteriores & 2,04 \\
\hline No sabe & 13,26 \\
\hline \multicolumn{2}{|l|}{ Alimentos más cariogénicos (que produce caries) para el infante } \\
\hline Leche materna & 4,08 \\
\hline Leche de fórmula (leche en polvo, entera o maternizada) & 3,06 \\
\hline Leche azucarada & 28,57 \\
\hline Bebida gaseosa* & 63,27 \\
\hline No sabe & 1,02 \\
\hline \multicolumn{2}{|l|}{ ¿Qué sucede cuando no es tratada una lesión de caries? } \\
\hline Se puede caer el diente & 5,1 \\
\hline La caries avanzará a través del esmalte hasta la dentina* & 55,1 \\
\hline Dependiendo del sistema inmunológico del infante, la caries se detendrá o avanzará & 13,27 \\
\hline La caries llegará a entrar al torrente sanguíneo & 4,08 \\
\hline Todas las anteriores & 21,43 \\
\hline No sabe & 1,02 \\
\hline \multicolumn{2}{|l|}{ Antibiótico de primera elección para tratar infecciones odontogénicas producidas por caries dental } \\
\hline Amoxicilina* & 90,82 \\
\hline Azitromicina & 5,1 \\
\hline Doxiciclina & 1,02 \\
\hline Penicilina & 1,02 \\
\hline Dicloxacilina & 2,04 \\
\hline * Respuesta correcta & \\
\hline
\end{tabular}


Tabla 3. Conocimientos sobre prevención e higiene bucal en niños. Médicos Residentes de Pediatría, Paraguay 2015

\begin{tabular}{|c|c|}
\hline Variables & $\%$ \\
\hline \multicolumn{2}{|l|}{ ¿Cuándo se debe iniciar la higiene oral del niño? } \\
\hline Tres meses & 10,2 \\
\hline Cuando el niño pueda sentarse y cooperar & 11,22 \\
\hline Cuando el infante sea consciente y comprenda la importancia de la higiene bucal & 15,31 \\
\hline Desde el nacimiento* & 14,29 \\
\hline Desde la erupción del primer diente & 46,94 \\
\hline No sabe & 2,04 \\
\hline \multicolumn{2}{|l|}{ Elementos de higiene bucal sería(n) el (los) más adecuado(s) para un infante de 9 meses } \\
\hline Gasa embebida con agua * & 28,57 \\
\hline Cepillo dental solo & 39,8 \\
\hline Cepillo y pasta dental* & 26,53 \\
\hline Cepillo y bicarbonato de sodio & 1,02 \\
\hline A esa edad todavía no es necesario realizarle higiene bucal & 1,02 \\
\hline No sabe & 3,06 \\
\hline \multicolumn{2}{|l|}{ ¿Cuándo se debe iniciar la fluorización en el infante? } \\
\hline Desde el nacimiento & 5,1 \\
\hline Cuando erupción el primer diente* & 10,2 \\
\hline A los 2 años & 21,43 \\
\hline A los 3 años, cuando han erupcionado todos los dientes temporales & 52,04 \\
\hline Ninguna de las anteriores & 5,1 \\
\hline No sabe & 6,13 \\
\hline \multicolumn{2}{|l|}{ Mejor forma de administrar flúor para niños } \\
\hline a. Flúor prenatal vía sistémica & 19,39 \\
\hline b. Fluorización del agua potable & 19,39 \\
\hline c. Flúor tópico en forma de barniz de flúor & 11,22 \\
\hline d. Flúor tópico en forma de gel & 26,53 \\
\hline e. Son correctos b y c* & 9,18 \\
\hline f. No sabe & 14,29 \\
\hline \multicolumn{2}{|l|}{ Edad ideal para realizar la primera consulta odontológica } \\
\hline Desde el nacimiento & 5,1 \\
\hline Entre los 6 y 12 meses* & 52,04 \\
\hline Cuando el niño pueda sentarse solo & 15,31 \\
\hline Cuando el niño pueda colaborar con el tratamiento odontológico & 14,29 \\
\hline No mayor de los 3 años & 11,22 \\
\hline No sabe & 2,04 \\
\hline
\end{tabular}


Tabla 4. Actitud acerca del papel del pediatra en la prevención de la caries dental. Médicos Residentes de Pediatría, Paraguay 2015

\begin{tabular}{|c|c|}
\hline Variables & $\%$ \\
\hline \multicolumn{2}{|c|}{ El pediatra debe ser el principal proveedor de información y consejo sobre salud oral de los niños } \\
\hline Totalmente de acuerdo & 70,41 \\
\hline De acuerdo & 25,51 \\
\hline Indiferente & 3,06 \\
\hline En desacuerdo & 0 \\
\hline Totalmente en desacuerdo & 1,02 \\
\hline \multicolumn{2}{|c|}{ El pediatra debe examinar los dientes y realizar el diagnóstico de caries dental en las consultas de control del niño sano } \\
\hline Totalmente de acuerdo & 63,27 \\
\hline De acuerdo & 32,65 \\
\hline Indiferente & 2,04 \\
\hline En desacuerdo & 2,04 \\
\hline Totalmente en desacuerdo & 0 \\
\hline \multicolumn{2}{|c|}{ Usted remitiría al infante donde el odontólogo solo cuando se observen lesiones cariosas } \\
\hline Totalmente de acuerdo & 60,2 \\
\hline De acuerdo & 20,41 \\
\hline Indiferente & 1,02 \\
\hline En desacuerdo & 13,27 \\
\hline Totalmente en desacuerdo & 5,1 \\
\hline \multicolumn{2}{|c|}{ ¿Usted examinaría los dientes a sus pacientes niños, buscando signos de caries? } \\
\hline Totalmente de acuerdo & 76,53 \\
\hline De acuerdo & 17,35 \\
\hline Indiferente & 3,06 \\
\hline En desacuerdo & 3,06 \\
\hline Totalmente en desacuerdo & 0 \\
\hline \multicolumn{2}{|c|}{ ¿Usted asesoría o brindaría consejo sobre prevención de caries a los padres de sus pacientes niños? } \\
\hline Totalmente de acuerdo & 77,55 \\
\hline De acuerdo & 20,41 \\
\hline Indiferente & 2,04 \\
\hline En desacuerdo & 0 \\
\hline Totalmente en desacuerdo & 0 \\
\hline \multicolumn{2}{|c|}{ Como parte de su práctica regular, ¿Usted referiría al paciente infante donde el odontólogo para consultas de prevención? } \\
\hline Totalmente de acuerdo & 81,63 \\
\hline De acuerdo & 18,37 \\
\hline Indiferente & 0 \\
\hline En desacuerdo & 0 \\
\hline Totalmente en desacuerdo & 0 \\
\hline
\end{tabular}




\section{Referencias bibliográficas}

1. González-Martínez F, Sánchez-Pedraza R, CarmonaArango L. Indicadores de riesgo para la caries dental en niños preescolares de la Boquilla, Cartagena. Rev Salud Pública. 2009;11(4):620-30.

2. Wulaerhan J, Abudureyimu A, Bao XL, Zhao J. Risk determinants associated with early childhood caries in Uygur children: a preschool-based cross-sectional study. BMC Oral Health. 2014;14:136.

3. Naidu R, Nunn J, Kelly A. Socio-behavioural factors and early childhood caries: a cross-sectional study of preschool children in central Trinidad. BMC Oral Health. 2013;13:30.

4. Sanabria-Castellanos CM, Suárez-Robles MA, Estrada-Montoya JH. Relación entre determinantes socioeconómicos, cobertura en salud y caries dental en veinte países. Rev Gerenc Polit Salud. 2015;14(28):161-89.

5. Aguilar-Ayala FJ, Duarte-Escobedo CG, Rejón-Peraza ME, Serrano-Pińa R, Pinzón-Te AL. Prevalencia de caries de la infancia temprana y factores de riesgo asociados. Acta Pediatr Mex. 2014;35(4):259-66.

6. González E, Pérez-Hinojosa S, Alarcón JA, Peñalver MA. Conocimiento de pediatras y padres andaluces sobre caries de aparición temprana. An Pediatr (Barc). 2015;82(1):19-26.

7. Achahui-Arauco P, Albinagorta MJ, Arauzo-Sinchez CJ, Cadenillas-Sueldo AM, Céspedes-Martínez GP, Cigüeñas-Raya EM, et al. Caries de Infancia temprana: diagnóstico e identificación de factores de riesgo. Odontol Pediatr. 2014;13(2):119-37.

8. Morrow JW, Keels MA, Hale KJ, Thomas HF, Davis MJ, Czerepak CS, et al. Preventive oral health intervention for pediatricians. Pediatrics. 2008;122(6):1387-94. DOI: $10.1542 /$ peds.2008-2577

9. Casamassimo PS, Thikkurissy S, Edelstein BL, Maiorini E. Beyond the $\mathrm{dmft}$ : the human and economic cost of early childhood caries. J Am Dent Assoc. 2009;140(6):650-7.

10. Duggal MS, Cameron A, Toumba J. Odontología pediátrica. México D.F: Manual Moderno; 2014.

11. Heilmann A, Tsakos G, Watt RG. Oral Health Over the Life Course. En: Burton-Jeangros C, Cullati S, Sacker A, Blane D, editores. A Life Course Perspective on Health Trajectories and Transitions [Internet]. Cham $(\mathrm{CH})$ : Springer; 2015 [citado 29 de junio de 2017]. Disponible en: http://www.ncbi.nlm.nih.gov/books/NBK385369/

12. Fernandes IB, Pereira TS, Souza DS, Ramos-Jorge J, Marques LS, Ramos-Jorge ML. Severity of Dental Caries and Quality of Life for Toddlers and Their Families. Pediatr Dent. 2017;39(2):118-23.

13. Martins-Júnior PA, Vieira-Andrade RG, Corrêa-Faria P, Oliveira-Ferreira F, Marques LS, Ramos-Jorge ML. Impact of early childhood caries on the oral health-related quality of life of preschool children and their parents. Caries Res. 2013;47(3):211-8. DOI: 10.1159/000345534

14. Jiménez JH. Formation of Human Resources in Pediatrics. Pediatr (Asunción). 2009;36(1):42-7.
15. Consejo Nacional de Residencias Médicas (CONAREM). Admisión por concurso a las residencias de especialidades troncales. Listado de unidades formadoras y plazas [Internet]. 2015 [citado 22 de setiembre de 2017]. Disponible en: http://www.conarem.ins.gov.py

16. Ministerio de Salud Pública y Bienestar Social. Reglamento General de la Comisión Nacional de Residencias Médicas y del Examen de Admisión [Internet]. 2013 [citado 22 de setiembre de 2017] . Disponible en: http:// www.conarem.ins.gov.py

17. Ministerio de Salud Pública y Bienestar Social. Regiones Sanitarias [Internet]. 2017. [citado 22 de setiembre de 2017]. Disponible en: http://www.mspbs.gov.py/dinavisa/regiones-sanitarias/

18. Rolón-Lara MC, Samudio M. Conocimiento, actitud y práctica de los médicos pediatras sobre factores preventivos de la salud oral en la primera infancia. Pediatr. (Asunción). 2014;41(3):191-200.

19. Hulley SB, Cummings SR, Newman TB. Diseño de estudios transversales y de cohortes. En: Diseño de investigaciones clínicas. 4ta ed. Barcelona: Wolters Kluwer Health; 2014.

20. Comisión Nacional de Residencias Médicas. Admisión por concurso a las residencias de especialidades troncales. Listado de unidades formadoras y plazas [Internet]. 2015. Disponible en: http://www.conarem.ins.gov.py

21. Ministerio de Salud Pública y Bienestar Social. Reglamento General de la Comisión Nacional de Residencias Médicas y del Examen de Admisión [Internet]. 2013. Disponible en: http://www.conarem.ins.gov.py

22. Rojas-Mayhuire PM. Nivel de conocimiento y actitud sobre caries de infancia temprana de los pediatras del Colegio Médico del Perú [Tesis de Licenciatura]. Universidad Nacional Mayor San Marcos; 2008 [consultado 13 de septiembre de 2016]. Disponible en: http://cybertesis.unmsm.edu.pe/handle/cybertesis/2178

23. Kumar P, Kumar P, Dixit A, Gupta V, Singh H, Sargaiyan V. Cross-Sectional Evaluation of Awareness of Prevention of Dental Caries Among General Pediatricians in Ghaziabad District, India. Ann Med Health Sci Res. 2014;4(3):302-6

24. Nassiri M, Karamali M. Interns' knowledge about pediatrics teeth and mouth health in Tehran educational hospitals, Iran, 2011. J Mazand Univ Med Sci. 2014;23(110):218-23.

25. Al Yousef Y, Damiano P, Weber-Gasparoni K, Qian F, Murph J, Nothwehr F. Medical students' child oral-health-related knowledge, practices and attitudes. Eur J Dent Educ. 2013;17(4):218-24. DOI: 10.1111/eje.12041

26. Long CM, Quinonez RB, Beil HA, Close K, Myers LP, Vann WF, et al. Pediatricians' assessments of caries risk and need for a dental evaluation in preschool aged children. BMC Pediatr. 2012;12:49. 